\title{
DEFINIZIONE DI UN METODO DI VERIFICA PER FORZE SISMICHE COMPLANARI ALLE PARETI DI EDIFICI AGRICOLI TRADIZIONALI
}

\author{
Giovanni Cascone, Claudia Arcidiacono
}

\section{PREMESSA}

\section{Foreword}

Il riuso e la riqualificazione funzionale dei fabbricati rurali tradizionali con struttura portante in muratura spesso richiedono interventi strutturali che siano al tempo stesso rispettosi dei caratteri architettonici degli edifici ed atti a garantirne, in relazione alle condizioni di fruizione, un sufficiente grado di sicurezza.

Nel caso di edifici rurali, si pone la necessità di definire metodi di verifica strutturale adeguati al loro specifico comportamento in condizioni sismiche. Ciò perché molti edifici agricoli d'esercizio - come ad esempio magazzini, granai, frantoi oleari, palmenti, stalle, cantine - presentano materiali edilizi, tecniche costruttive e tipologie strutturali che ne determinano un carattere "specialistico"e che, in genere, non consentono l'applicazione dei metodi di verifica sismica solitamente adottati per gli edifici in muratura. Tali metodi, infatti, hanno un ben delimitato campo di validità e sono modellati su strutture tipiche degli edifici residenziali urbani i quali, generalmente, sono costituiti da più piani fuori terra, presentano una limitata superficie in pianta, sono fondati su un'unica giacitura orizzontale, hanno spessori non particolarmente elevati delle murature perimetrali e solai rigidi efficacemente connessi a quest'ultime e presentano generale regolarità nell'allineamento delle aperture.

Fabbricati agricoli d'esercizio quali quelli soprelencati, invece, solitamente si presentano come scatole murarie monoconnesse, essendo costituiti da un unico ambiente ad una sola elevazione e senza partizioni interne, privi di impalcati rigidi, con murature in pietrame grossolanamente squadrato e strutture lignee di copertura prive di rigidezza nei piani di falda e collegate ai muri d'ambito da vincoli monolateri sostan-

Memoria presentata il 04.05.2006; accettata il 22.02.2007

Prof. Ing. Giovanni Cascone, Professore ordinario, Dipartimento di Ingegneria Agraria dell'Università degli Studi di Catania.

Prof. Ing. Claudia ARCidiacono, Professore associato, Dipartimento di Ingegneria Agraria dell'Università degli Studi di Catania. zialmente inefficaci a limitarne gli spostamenti orizzontali, essendo tale compito esclusivamente demandato all'attrito tra le superfici a contatto. L'assenza di orizzontamenti rigidi, in particolare, non consente di basare la verifica strutturale sull'ipotesi di uguali spostamenti orizzontali di piano, che trova pressoché generale applicazione nei metodi di calcolo sismico oggi più diffusi. Non potendo attribuire un idoneo comportamento tridimensionale alla scatola muraria, lo schema strutturale più adeguato a rappresentare il reale comportamento dell'edificio è quello che considera ciascuna parete indipendente dalle altre, soggetta ai carichi verticali e alle forze sismiche orizzontali che le competono.

Edifici agricoli tradizionali del tipo descritto, sotto l'azione del sisma presentano come meccanismo di collasso più probabile il ribaltamento verso l'esterno delle pareti perimetrali. In altra sede [3] si è mostrato che la verifica strutturale rispetto ad azioni sismiche ortogonali alla parete, applicata ad alcuni edifici agricoli caratteristici della regione etnea (zona sismica di $2^{\text {a }}$ categoria) nell'ambito delle ipotesi semplificative ammesse dalla normativa vigente (legame costitutivo lineare della muratura e sezioni interamente reagenti), porta in genere a risultati negativi.

Pur tuttavia, le osservazioni condotte su fabbricati agricoli presenti nel territorio etneo dotati di caratteristiche analoghe a quelle degli edifici sottoposti a verifica, in occasione di recenti eventi sismici, non hanno evidenziato collassi strutturali per ribaltamento o la presenza di dissesti imputabili a meccanismi di rotazione al piede dei muri perimetrali. Ciò induce a considerare l'esito negativo del calcolo di verifica come dovuto anche ad una insufficiente approssimazione dell'effettivo comportamento strutturale da parte del modello di calcolo. Occorrerebbe, pertanto, discostarsi dalle ipotesi semplificative consentite dalla normativa e sviluppare modelli che siano più aderenti alle effettive caratteristiche reologiche delle murature, tengano conto della non linearità del legame costitutivo della muratura e della mancanza di resistenza a trazione del materiale. In ogni caso appare opportuno procedere ad interventi migliorativi del comportamento strutturale, tendenti a contrastare la rotazione verso 
l'esterno dei muri perimetrali, soprattutto attraverso l'introduzione di vincoli più efficaci in testa alle murature $[3,4]$.

Se la verifica al ribaltamento è soddisfatta, o se, comunque, si adottano interventi che efficacemente impediscano il meccanismo di rotazione al piede della parete, allora occorre prendere in considerazione il secondo più probabile modo di collasso dovuto all'azione delle forze sismiche complanari alla parete. Il presente lavoro intende proporre un metodo di calcolo di verifica sismica nel caso di azioni complanari alla parete specificamente dedicato alle tipologie "specialistiche" di fabbricati agricoli sopra citate.

\section{METODO DI CALCOLO}

\section{Method formulation}

Nel metodo di calcolo proposto la parete viene considerata composta dai maschi verticali e da una fascia di piano orizzontale. I primi sono costituiti dagli elementi verticali di muratura compresi tra le aperture mentre la fascia di piano è costituita dalla parte di muratura soprastante la linea ideale che congiunge gli architravi delle aperture. Il metodo prevede la schematizzazione della parete muraria come telaio a nodi spostabili (ipotesi "shear-type") rappresentando i maschi come ritti e la fascia di piano come un traverso dotato di rigidezza infinita sotto l'aspetto deformativo ma non infinitamente resistente [11]. Nella prima fase del calcolo viene valutata la resistenza ultima del telaio nell'ipotesi di comportamento elasto-plastico dei ritti mentre nella seconda fase viene condotta l'analisi delle sollecitazioni indotte nelle campate costituenti il traverso al fine di verificare se la fascia di piano, nel complesso, soddisfa l'ipotesi di rigidezza infinita impedendo o, quanto meno, limitando efficacemente le rotazioni dei nodi d'estremità dei ritti.

\subsection{DETERMINAZIONE DELLA RESISTENZA MASSIMA DELLA PARETE}

\subsection{Determination of the maximum wall resistance}

Detti $i$ il generico passo incrementale, NP il numero totale dei passi incrementali, $k$ il generico maschio ed NM il numero complessivo dei maschi costituenti la parete, il metodo adottato per la determinazione della resistenza ultima della parete e per la costruzione della relativa curva caratteristica può essere riepilogato nel seguente procedimento:

1) Si traccia la curva caratteristica di ciascun maschio murario costituente la parete tramite il calcolo delle rigidezze $\left(\mathrm{K}_{1}\right.$ e $\left.\mathrm{K}_{2}\right)$, degli spostamenti $\left(\delta_{1}, \delta_{2} \mathrm{e}\right.$ $\left.\delta_{3}\right)$ e delle forze orizzontali massime $\left(\mathrm{V}_{1}, \mathrm{~V}_{\mathrm{u}}\right)$ nelle ipotesi di rottura per fessurazione diagonale, per scorrimento orizzontale lungo i letti di malta e per flessione $[3 ; 6 ; 8 ; 10]$ (fig. 1 ).

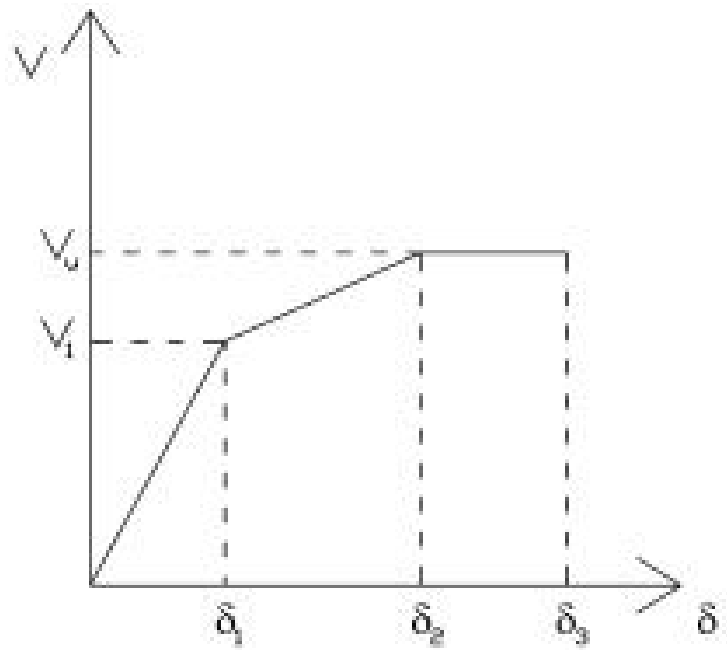

Fig. 1 - Curva caratteristica dei maschi murari.

Fig. 1 - Rheologic curve of wall panels.

2) Si sceglie una coppia di valori $\mathrm{F}_{0}, \delta_{0}$ di primo tentativo per la forza orizzontale e per il corrispondente spostamento, con la sola precauzione di adottare una forza $\mathrm{F}_{0}$ molto minore della forza sismica $\mathrm{F}_{\mathrm{s}}$ valutata secondo la normativa vigente $[8 ; 12]$.

3) $\mathrm{Si}$ adotta un opportuno incremento $\Delta \mathrm{F}_{\mathrm{i}}$ della forza orizzontale $\mathrm{F}_{\mathrm{i}}=\mathrm{F}_{\mathrm{i}-1}+\Delta \mathrm{F}_{\mathrm{i}}$, variabile al crescere del numero dei passi incrementali in modo da migliorare l'approssimazione nella valutazione della resistenza ultima $\mathrm{F}_{\mathrm{u}}$ della parete. È opportuno, inoltre, che tale incremento sia fissato in modo che il valore della forza orizzontale $\mathrm{F}_{\mathrm{i}}$ in uno dei passi incrementali coincida con l'intensità della forza sismica $\mathrm{F}_{\mathrm{s}}$, al fine di conoscere lo stato di sollecitazione nei ritti e nel traverso proprio in corrispondenza del valore della forza sismica dettata dalla normativa vigente. I valori di $\Delta \mathrm{F}_{\mathrm{i}}$ vanno scelti in modo che la forza $\mathrm{F}_{\mathrm{i}}$ risulti monotona strettamente crescente.

4) Si determina la rigidezza del traverso $K_{i}$, relativa al generico passo incrementale $i$, come somma della rigidezza $\mathrm{K}_{\mathrm{i}, \mathrm{k}}$ di ciascun maschio corrispondente allo spostamento $\delta_{\mathrm{i}-1}$ determinato nel passo incrementale precedente:

$$
K_{i}=\sum_{i=1}^{N M} K_{i h}
$$

5) Viene avviato un ciclo iterativo interno con contatore $j$ per la individuazione dello spostamento $\delta_{i}$ corrispondente alla forza $F_{i}$. A tal fine si calcola, innanzitutto, l'incremento di spostamento:

$$
\Delta \delta_{i}=\frac{\Delta F_{i}}{K_{i}}
$$

e lo spostamento totale della parete relativo alla iterazione $j$ :

$$
\delta_{i}^{j}=\delta_{i}^{j-1}+\Delta \delta_{i}^{j} .
$$

6) In corrispondenza dello spostamento totale $\delta_{i}^{j}$, si individuano $\mathrm{i}$ valori delle forze reattive dei maschi murari sulle rispettive curve caratteristiche: 


$$
\mathrm{V}_{\mathrm{i}, \mathrm{k}}^{\mathrm{j}}=\mathrm{K}_{\mathrm{i}, \mathrm{k}}^{\mathrm{j}} \cdot \delta_{\mathrm{i}}^{\mathrm{j}}
$$

e la forza reattiva totale della parete:

$$
v_{i}^{j}=\sum_{k=1}^{N M} v_{i k}^{j}
$$

Si calcola il residuo della forza reattiva $\Delta \mathrm{V}_{\mathrm{i}}^{\mathrm{j}}=\mathrm{F}_{\mathrm{i}}-$ $\mathrm{V}_{\mathrm{i}}^{\mathrm{j}}$ e si controlla la condizione:

$$
\left|\frac{\Delta \mathrm{V}_{\mathrm{i}}^{\mathrm{j}}}{\Delta \mathrm{F}_{\mathrm{i}}}\right|<\boldsymbol{\varepsilon}
$$

essendo $\varepsilon$ il limite di approssimazione accettabile prefissato. Se la verifica non è soddisfatta si procede ad una ulteriore iterazione. A tal fine viene aggiornato il valore della rigidezza alla traslazione del traverso:

$$
K_{i}^{j}=\frac{V_{i}^{j}}{\delta !}
$$

e valutato l'incremento di spostamento:

$$
\Delta \delta_{i}^{j+1}=\frac{\Delta V_{i}^{j}}{K_{i}^{j}}
$$

che determina il nuovo spostamento totale: $\delta_{i}^{\mathrm{j}+1}=\delta_{i}^{\mathrm{j}}+$ $\Delta \delta_{i}^{j+1}$. Nel caso in cui $\Delta \mathrm{V}_{i}^{\mathrm{j}}$ è negativo risulterà $\Delta \delta_{i}^{j+1}<0$.

I valori $\mathrm{F}_{\mathrm{i}}, \delta_{\mathrm{i}}$ relativi alla iterazione in cui è soddisfatto il controllo su e rappresentano le coordinate del punto della curva caratteristica del traverso corrispondente all'incremento di carico $\Delta \mathrm{F}_{\mathrm{i}}$. I valori delle forze $\mathrm{V}_{\mathrm{i}, \mathrm{k}}^{\mathrm{j}}$ che soddisfano la condizione suddetta consentono di valutare le azioni taglianti nelle sezioni d'estremità delle singole campate del traverso.

7) Si incrementa di una unità il contatore $i$, si impone un nuovo incremento $\Delta \mathrm{F}_{\mathrm{i}}$ alla forza orizzontale e si ripercorrono i passi a partire dal 4) utilizzando lo spostamento $\delta_{i-1}$ ottenuto nel passo incrementale precedente.

Allorché per un dato valore di $\mathrm{F}_{\mathrm{i}}$ in ogni iterazione successiva del ciclo interno risulta $\Delta \delta_{i}^{j}$ sempre positivo, il $\delta_{i}^{\mathrm{j}}$ cresce fino a superare il $\delta_{3}$ di tutti i maschi e si ottiene per ciascuno di essi $\mathrm{V}_{\mathrm{i}, \mathrm{k}}^{\mathrm{j}}=0$. Tale condizione determina la fine della costruzione della curva caratteristica del traverso e fornisce la resistenza massima $F_{u}$ offerta dalla parete pari all'ultimo valore di $F_{i}$ per il quale si ha $\mathrm{V}_{\mathrm{i}} \geq \mathrm{F}_{\mathrm{i}}$. Essa rappresenta il valore oltre il quale le risorse di resistenza dei maschi non sono sufficienti a contrastare la forza orizzontale esterna e si perviene al collasso della parete. La coppia di valori $\mathrm{F}_{\mathrm{u}}$ e $\delta_{\mathrm{u}}$ rappresenta le coordinate dell'ultimo punto della curva caratteristica del traverso.

La verifica della parete è soddisfatta se si realizza la condizione seguente:

$$
v=\frac{F_{\mathrm{u}}}{F_{\mathrm{s}}} \geq 1
$$

in cui n è il coefficiente di sicurezza della parete rispetto alla forza sismica prevista dalla normativa vigente.

Il diagramma di flusso del modello proposto è riportato in fig. 2 .

Differentemente da quanto previsto nel metodo POR, nel metodo qui proposto il processo di accumu- lo dei contributi resistenti di ciascun maschio non si arresta quando uno dei maschi raggiunge per primo il valore dello spostamento ultimo $\delta_{3}$. In tal modo possono essere più appropriatamente valutati i valori della resistenza ultima $\mathrm{F}_{\mathrm{u}}$ di pareti i cui maschi murari presentano dimensioni molto diverse e, di conseguenza, curve caratteristiche il cui tratto orizzontale corrisponde ad intervalli di spostamento differenti. Tale proprietà rende il metodo particolarmente idoneo allo studio delle pareti degli edifici agricoli tradizionali che, a differenza degli edifici urbani in muratura portante, presentano spesso forti asimmetrie.

I limiti dei modelli basati sull'ipotesi di "meccanismo di piano" che eseguono analisi non lineari tagliospostamento separatamente per ogni interpiano, evidenziati da alcuni Autori [9] possono ritenersi superati in virtù della semplicità strutturale degli edifici analizzati. Infatti, poiché l'osservazione che i fenomeni di fessurazione o di rottura degli elementi orizzontali di accoppiamento (fasce murarie e/o cordoli in c.a.) possono essere valutati in maniera sufficientemente accurata solamente con un'analisi globale della parete multipiano o dell'edificio viene superata nel caso degli edifici considerati, in virtù della presenza di un'unica elevazione.

\subsection{VERIFICA DELLA FASCIA DI PIANO}

\subsection{Safety verification of floor strip}

Il comportamento delle fasce non è stato studiato, soprattutto sperimentalmente, con la stessa attenzione dedicata ai maschi murari. La funzione strutturale delle fasce è tutt'altro che secondaria per le pareti multipiano poiché fornendo l'accoppiamento tra i montanti murari, possono influenzarne il meccanismo di risposta. Tale influenza, tuttavia, è tanto più grande quanto maggiore è il numero di piani di un edificio [9].

La presenza di catene o cordoli assicura la resistenza flessionale della fascia. Questi opponendosi alla dilatazione globale della parete in senso orizzontale, generano un incremento di compressione nelle fasce che aumenta la resistenza a flessione delle stesse [9]. Nel seguito, infatti, verrà evidenziato che tale condizione viene tenuta in considerazione nel calcolo delle grandezze resistenti secondo normativa.

Indicando con $i$ il generico maschio murario e con $j$ la generica campata della fascia di piano, per quest'ultima è possibile calcolare lo sforzo di taglio $\mathrm{T}_{\mathrm{j}}$ nelle sezioni d'estremità ed il momento flettente $\mathrm{M}_{\mathrm{j}}$ nella sezione di mezzeria mediante le relazioni [2]:

$$
\begin{gathered}
\mathrm{T}_{\mathrm{j}}=\frac{\rho_{\mathrm{jsx}} \mathrm{v}_{\mathrm{i}}\left(\mathrm{r}_{\mathrm{i}}+\mathrm{h}_{\mathrm{i}} / 2\right)+\rho_{\mathrm{jdx}} \mathrm{v}_{\mathrm{i}+1}\left(\mathrm{r}_{\mathrm{i}}+\mathrm{h}_{\mathrm{i}+1} / 2\right)}{\mathrm{C}_{\mathrm{j}}} ; \\
\mathrm{M}_{\mathrm{j}}=\frac{\mathrm{T}_{\mathrm{j}} \mathrm{b}_{\mathrm{j}}}{2}
\end{gathered}
$$

essendo $\mathrm{V}_{\mathrm{i}}$ lo sforzo tagliante nell'i-esimo maschio murario, $h_{i}$ l'altezza del maschio i-esimo, $r_{i}$ la semialtezza della fascia i-esima, $\rho_{j \mathrm{sx}}$ e $\rho_{\mathrm{jDX}}$ i coefficienti di 


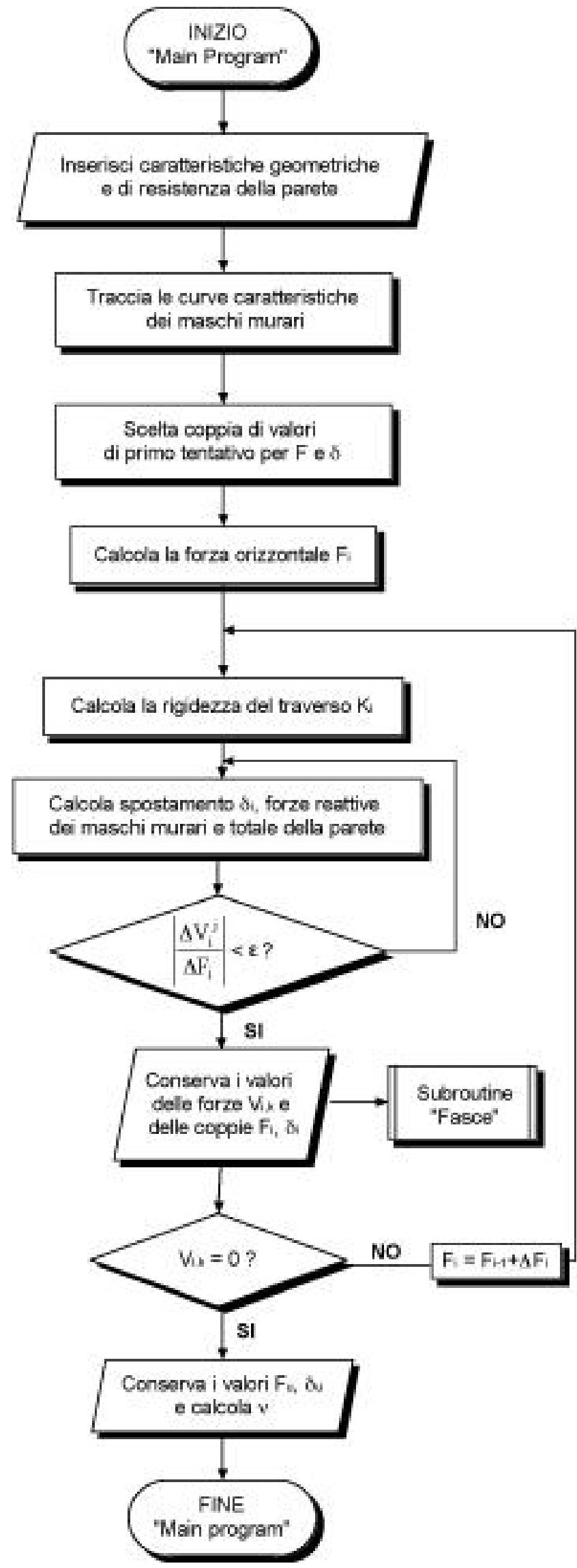

Fig. 2 - Diagramma di flusso del modello matematico proposto. Fig. 2 - Flowchart of the proposed mathematical model.

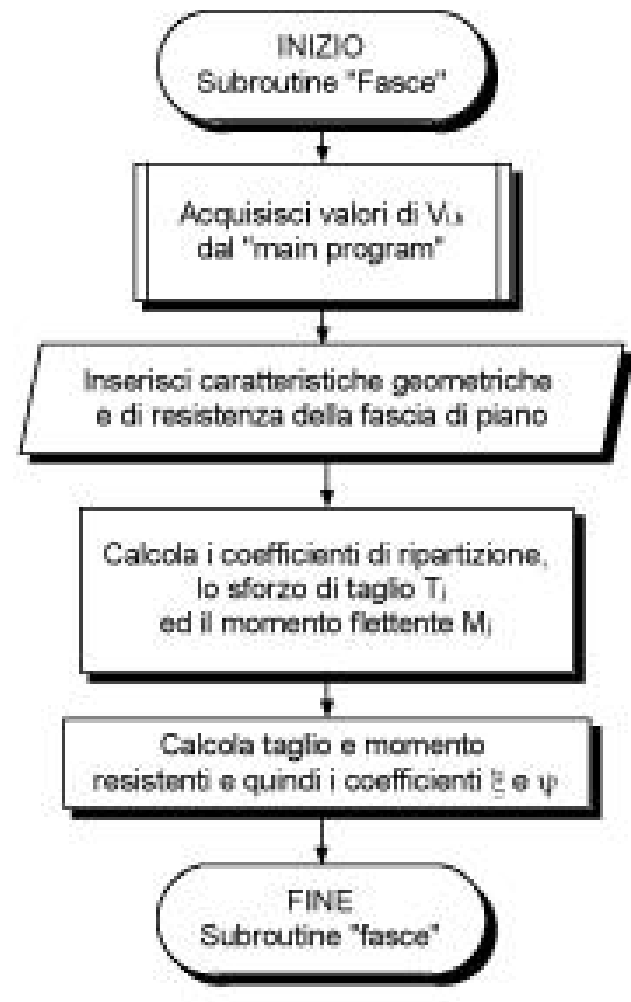

ripartizione, ricavati al successivo paragrafo $2.3, \ell_{\mathrm{j}}$ l'interasse tra due ritti successivi, $b_{j}$ la larghezza dell'apertura j-esima.

La verifica di resistenza della generica campata $j$ della fascia di piano è soddisfatta se si realizzano le condizioni seguenti:

$$
\xi=\frac{T_{R_{j}}}{T_{j}} \geq 1 \quad \psi=\frac{M_{R_{j}}}{M_{j}} \geq 1
$$

dove $T_{R j}$ ed $M_{R j}$ sono, rispettivamente, il taglio ed il momento resistenti.

Il taglio resistente è dato dalla seguente relazione:

$$
\mathrm{T}_{\mathrm{Rj}}=\mathrm{s}_{\mathrm{j}} \mathrm{a}_{\mathrm{j}} \tau_{\mathrm{kf}}
$$

in cui $\tau_{\mathrm{kf}}$ è la resistenza caratteristica alla fessurazione diagonale della fascia di piano, $a_{j}$ ed $s_{j}$ sono rispettivamente l'altezza e lo spessore della j-esima campata della fascia di piano.

Il momento resistente viene ricavato mediante la relazione:

$$
\mathrm{M}_{\mathrm{Rj}}=\sigma_{\mathrm{fp}} \mathrm{W}_{\mathrm{cj}}
$$

in cui $\sigma_{\mathrm{fp}}$ è la resistenza caratteristica a flessione della muratura costituente la fascia di piano mentre $\mathrm{W}_{\mathrm{cj}}$ è il modulo di resistenza della sezione rispetto all'asse neutro. Poiché la sezione è omogenea:

$$
M_{R_{j}}=\frac{1}{6} s_{j} a_{j}^{2} \sigma_{6}
$$

Il taglio ed il momento resistenti sono stati calcolati anche secondo quanto descritto al punto 5.4.6.2.5 della normativa vigente [8]. 


\subsection{CALCOLO DEI COEFFICIENTI DI RIPARTIZIONE}

\subsection{Determination of the distribution coefficients}

Se le pareti sono dotate di simmetria geometrica, come spesso avviene nell'edilizia urbana, per l'equilibrio dei nodi tra $\mathrm{i}$ ritti ed il traverso i momenti alle estremità delle campate risultano uguali [2]. Se, invece, come generalmente si riscontra negli edifici agricoli tradizionali, le pareti sono asimmetriche, i momenti nei nodi si distribuiscono in dipendenza della rigidezza rotazionale degli elementi afferenti ai nodi stessi. Detti $M_{1}$ ed $M_{2}$ i momenti alle estremità delle campate che convergono in un nodo e $\rho_{1}$ e $\rho_{2}$ i rispettivi coefficienti di ripartizione che consentono di esprimere $\mathrm{M}_{1}$ ed $\mathrm{M}_{2}$ in funzione del valore $\mathrm{M}$ del momento nella sezione estrema del ritto, per l'equilibrio del nodo si può scrivere:

$$
M=M_{1}+M_{2}=\rho_{1} M+\rho_{2} M
$$

Essendo $\mathrm{R}$ la rigidezza rotazionale della campata e $\phi$ la rotazione del nodo, si ha:

$$
M_{1}=R_{1} \phi \quad M_{2}=R_{2} \phi
$$

Da tali relazioni si ricava:

$$
\rho_{1}=\frac{R_{1}}{R_{1}+R_{2}} \quad \rho_{2}=\frac{R_{1}}{R_{1}+R_{2}}
$$

L'equilibrio alla rotazione delle campate $(\mathrm{j}-1)$ e (j) comprese tra $i$ tre ritti (i-1), (i) ed (i+1) facenti parte di un telaio costituito da $n$ ritti, è espresso dalla coppia di equazioni:

$$
\begin{aligned}
\mathrm{T}_{j+1} \ell_{j-1} & =\mathrm{M}_{j \mathrm{hax}}+\mathrm{M}_{\text {Hia }} \\
\mathrm{T}_{\mathrm{j}} \ell_{j} & =\mathrm{M}_{\mathrm{jx}}+\mathrm{M}_{\mathrm{jix}}
\end{aligned}
$$

che, tenendo conto della condizione di equilibrio al nodo prima ricavata, assumono la forma seguente:

$$
\begin{aligned}
\mathrm{T}_{j-1} \ell_{j-1} & =\rho_{j-\mathrm{bx}_{\mathrm{x}}} \mathrm{M}_{i-1}+\rho_{j-\mathrm{h}_{\mathrm{x}}} \mathrm{M}_{i} \\
\mathrm{~T}_{\mathrm{j}} \epsilon_{\mathrm{j}} & =\rho_{\mathrm{jx}} \mathrm{M}_{\mathrm{i}}+\rho_{\mathrm{jox}} \mathrm{M}_{\mathrm{i}+1}
\end{aligned}
$$

Poiché in un telaio shear-type il valore del momento $\mathrm{M}$ alla sommità di ciascun ritto risulta legato alla sollecitazione di taglio $\mathrm{V}$ in tale sezione ed all'altezza $\mathrm{h}$ del ritto tramite la relazione:

$$
\mathrm{M}=\frac{\mathrm{Vh}}{2}
$$

La coppia di equazioni precedenti può essere riscritta nel modo seguente:

$$
\begin{aligned}
T_{j+1} \ell_{j-1} & =\rho_{j-s s} \frac{V_{i-1} b_{i-1}}{2}+\rho_{j-l i x s} \frac{V_{i} h_{i}}{2} \\
T_{j} \ell_{j} & =\rho_{b s} \frac{V_{i} h_{i}}{2}+\rho_{h x} \frac{V_{i+1} h_{i+1}}{2}
\end{aligned}
$$

Se i nodi (i-1) ed (i+1) sono il primo e l'ultimo nodo del traverso, risulta: $\rho_{\mathrm{j}-1_{\mathrm{Sx}}}=\rho_{\mathrm{ipx}}=1$ mentre per $\mathrm{i}$ generici nodi interni (i) ed (i+1) appartenenti alla campata j-esima si ha rispettivamente:

$$
\begin{aligned}
& \rho_{j s}=\frac{R_{j s s}}{\left(R_{j_{s x}}+R_{j-I_{m s}}\right)}
\end{aligned}
$$

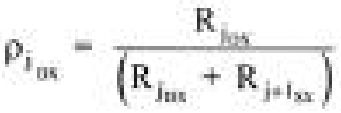

dove $R_{j-1}$ e $R_{j_{D X}}$ rappresentano, rispettivamente, le rigidezze rotazionali nel nodo destro delle campate $(\mathrm{j}-$ 1) e (j) del traverso, mentre $R_{j_{s x}}$ e $R_{j+1_{s x}}$ sono, rispettivamente, le rigidezze rotazionali nel nodo sinistro delle campate $(\mathrm{j})$ e $(\mathrm{j}+1)$ del traverso.

In generale, la rigidezza rotazionale di una trave appartenente ad un telaio dipende dalle sue dimensioni, dalle caratteristiche geometriche del telaio, dalle rigidezze degli altri elementi strutturali e dalle condizioni di vincolo esterno [14]. Nel presente lavoro, per semplicità di trattazione e compatibilmente con i livelli di approssimazione attesi, si è assunto che la rigidezza rotazionale in un nodo dipenda esclusivamente dalle caratteristiche geometriche della campata considerata e della campata adiacente al nodo in esame. Si ha, pertanto:

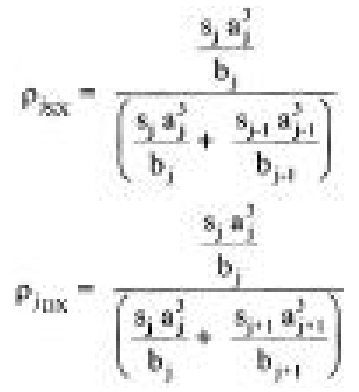

Se la fascia di piano fosse costituita da campate aventi dimensioni costanti, entrambi i coefficienti di ripartizione valutati con le formule precedenti risulterebbero pari a 0,5 .

\section{ANALISI DEI RISULTATI E DISCUSSIONE}

\section{Results analysis and discussion}

L'applicabilità del metodo di calcolo proposto è stata verificata studiando il comportamento statico di diverse pareti al variare di alcuni dei parametri geometrici avendo fissato le caratteristiche di resistenza della muratura.

Sono state preliminarmente analizzate pareti regolari e uniformi costituite da maschi e fasce di piano tutti di uguali dimensioni e aventi aperture identiche (tutte porte o tutte finestre di uguali dimensioni).

Successivamente, sono state analizzate le tipologie più frequentemente riscontrate per gli edifici agricoli d'esercizio tradizionali dell'area etnea [13] e sono stati conseguentemente individuati i tre principali schemi di pareti portanti in essi ricorrenti (fig. 3).

Nel modello proposto i parametri geometrici che maggiormente influenzano la risposta di una parete all'azione sismica sono lo spessore delle murature t, la larghezza dei maschi murari $\mathrm{D}_{\mathrm{m}}$, l'altezza delle fa- 
sce di piano. Pertanto, nei calcoli svolti utilizzando il modello proposto, lo spessore delle murature varia tra $0,3 \mathrm{~m}$ (maggiore di $0,24 \mathrm{~m}$, indicato nel punto 5.4.4 della normativa) e $1,1 \mathrm{~m}$; la larghezza dei maschi varia tra $1 \mathrm{~m}$ (come indicato dalla normativa per i maschi di bordo) e $6 \mathrm{~m}$; l'altezza della fascia di piano varia tra 0,3 e $2 \mathrm{~m}$ ovvero $2,5 \mathrm{~m}$ a seconda delle tipologie riscontrate. Con tali assunzioni è anche verificato che la snellezza convenzionale, definita dalla norma $[8 ; 12]$ come rapporto tra la lunghezza libera d'inflessione e lo spessore del maschio murario, si mantiene inferiore a 20.

La snellezza dei maschi murari, definita invece come rapporto tra l'altezza e la larghezza del maschio stesso, rappresenta il parametro dimensionale più significativo al fine di individuare il modo di collasso dei maschi murari costituenti le pareti. Essa, infatti, influisce in maniera determinante sulla forma delle curve caratteristiche dei maschi e, pertanto, sul comportamento statico da essi offerto al variare delle forze agenti.

Mentre nella vigente normativa [8;12] tale parametro, seppur indispensabile alla definizione delle curve reologiche, non è richiamato, al punto C.5.2 l) della normativa precedentemente in vigore [7] il valore limite della snellezza al di sopra del quale il maschio murario raggiunge il collasso e non dà più alcun contributo alla resistenza della parete veniva fissato pari a 3. Nel metodo adottato nel presente studio, invece, viene fissato un valore limite della snellezza pari a 1,5 , come indicato in precedenti studi [6], superato il quale il maschio continua a dare il proprio contributo
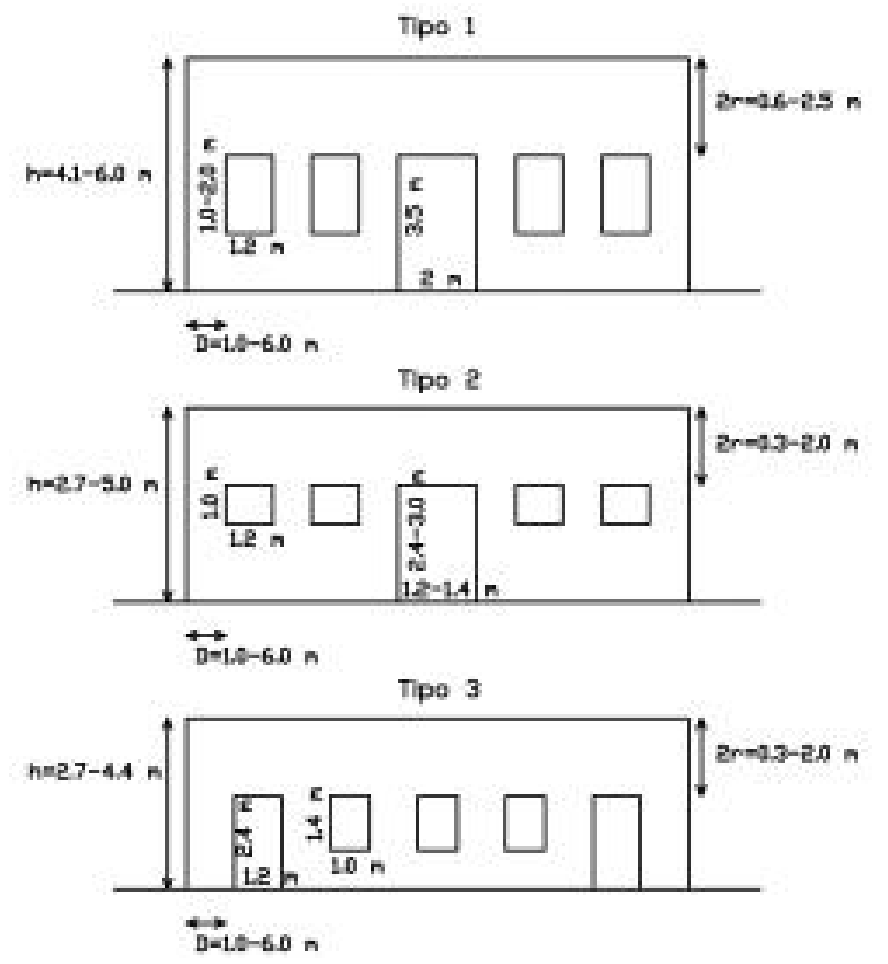

Fig. 3 - Principali schemi di pareti di fabbricati agricoli d'esercizio tradizionali.

Fig. 3 - Main analyzed schemes of traditional rural buildings walls. alla resistenza totale della parete. In tale caso, il maschio è sufficientemente snello da essere considerato un solido alla De St. Venant (ipotesi di conservazione delle sezioni piane e di materiale omogeneo e isotropo) e quindi il suo comportamento statico può essere assimilato a quello di una trave. Soltanto sotto tali ipotesi può essere calcolata la forza orizzontale resistente a flessione $\mathrm{V}_{\mathrm{u} \text { flex }}$ che risulta sempre minore della forza orizzontale resistente per fessurazione diagonale $\mathrm{V}_{\mathrm{u} \text {,iag }}$ e di quella per scorrimento orizzontale lungo i letti di malta $\mathrm{V}_{\mathrm{u} \text {,oriz }}$ [3]. Si osserva, inoltre, che adottando il valore limite della snellezza pari a 3 sulla base delle indicazioni dedotte dalla norma, per pareti che presentano uniformità strutturale il metodo proposto conduce a risultati meno cautelativi rispetto a quelli che si ottengono fissando un valore della snellezza limite pari a 1,5.

Inoltre, la relazione di $\mathrm{V}_{\mathrm{u}, \text { oriz }}$ adottata nel presente lavoro [6] determina valori sempre minori rispetto a quelli ottenuti con la relazione di $\mathrm{V}_{\mathrm{u}, \text { oriz }}$ proposta dalla norma [8;12]. Quest'ultima, in particolare, determina valori maggiori per la resistenza massima $F_{u}$ offerta dalla parete e quindi più elevati coefficienti di sicurezza $v$ della parete rispetto alla forza sismica, ma anche maggiori sollecitazioni di taglio trasmesse dai maschi alle fasce e, quindi, un eccessivo rischio di superamento della resistenza offerta dalle fasce.

La coppia di valori $\sigma_{k}$ e $\tau_{k}$, che rappresentano, rispettivamente, la resistenza a compressione e a taglio delle murature costituenti le pareti, sono quelle indicate nel tab. 1 dell'art. 10 delle istruzioni emanate in forza della legge $n^{\circ} 219 / 81$ [10] per murature in pietrame grossolanamente squadrato e bene organizzato. Tali valori risultano in buon accordo con quelli riscontrati sperimentalmente su provini di murature tipiche della zona etnea $[1 ; 5 ; 9]$.

Il carico permanente considerato per l'applicazione del modello proposto nel presente lavoro è dovuto al peso proprio della metà superiore del muro ed al peso proprio della copertura con struttura portante in legno e manto in coppi e canali tipica delle costruzioni rurali tradizionali mentre il carico accidentale è dovuto al peso della neve sulla copertura [8]. L'ulteriore sovraccarico accidentale è pari a $100 \mathrm{kgm}^{-1}$ secondo la normativa vigente [8]. L'azione del vento è stata trascurata nelle combinazioni di carico considerate per le elaborazioni svolte in quanto da apposite valutazioni è emerso che l'azione complanare alla parete esercitata dal vento è sempre trascurabile rispetto a quella sismica.

È stato verificato che tra le combinazioni dei carichi previste dalla normativa [8], prendendo a turno una delle azioni variabili come dominante, la più gravosa è quella in cui è dominante il sovraccarico in combinazione con la forza sismica e con la neve, anche rispetto a situazioni di solo carico permanente in assenza di ogni altra azione. Tale combinazione determina, infatti, le massime forze sismiche $\mathrm{F}_{\mathrm{s}}$ ed $\mathrm{i}$ minimi valori del coefficiente di sicurezza $v$.

Nelle prime elaborazioni eseguite è stato analizzato 
il comportamento di pareti regolari in funzione di variazioni della larghezza dei maschi $\mathrm{D}_{\mathrm{m}}$ (e quindi della loro snellezza), dell'altezza delle aperture $h_{f}$, della semi-altezza $r$ della fascia di piano e dello spessore $t$ della muratura.

In particolare, le elaborazioni per una parete dotata di uniformità strutturale, costituita da maschi di altezza 3,5 $\mathrm{m}$ e fasce di altezza $60 \mathrm{~cm}$, mostrano che, per $\mathrm{t} \leq 30 \mathrm{~cm}$, la parete non supera la verifica sismica quando tutti i maschi hanno snellezza maggiore di 2,8 . Invece, per spessori più elevati $(\mathrm{t}>70 \mathrm{~cm})$ la snellezza può raggiungere anche il valore di 3,5 senza che la parete arrivi al collasso.

In generale, dalle elaborazioni svolte è emerso che aumentando lo spessore della parete si ottengono forze ultime $\mathrm{F}_{\mathrm{u}}$ maggiori ma anche incrementi del carico permanente che si riflettono nell'aumento del peso sismico $\mathrm{W}_{\text {sism }}$ e quindi della forza sismica $\mathrm{F}_{\mathrm{s}}$. Dunque, un miglioramento della resistenza complessiva dei maschi costituenti la parete e della resistenza delle fasce di piano si ottiene aumentandone lo spessore, ma il corrispondente aumento della forza resistente ultima dei maschi $\mathrm{V}_{\mathrm{u}}$ determina incrementi più contenuti del coefficiente di sicurezza $v$ della parete rispetto a quelli che si ottengono per i coefficienti di sicurezza $\xi$ e $\psi$ delle fasce di piano. Infatti, dalle elaborazioni compiute, è risultato che raddoppiando lo spessore delle pareti l'incremento di $v$ è dell' $8-13 \%$ mentre l'incremento dei coefficienti di sicurezza $\xi$ e $\psi$ di ciascuna campata della fascia di piano è tra il $16 \%$ ed il $21 \%$.

Il consolidamento della muratura mediante iniezioni di cemento ovvero mediante due lastre di calcestruzzo armato, pur determinando un incremento dei valori di $\sigma_{k}$ e $\tau_{k}$ secondo quanto indicato dalla norma [10] e quindi $\mathrm{V}_{\mathrm{u}}$ maggiori, non sempre è sufficiente a incrementare il coefficiente di sicurezza $v$ e la resistenza delle fasce in modo tale da evitare il collasso della parete. Ad esempio, nella parete avente maschi uguali di altezza $3,5 \mathrm{~m}$, il consolidamento permette di evitare il crollo per spessori della muratura $t \geq 50 \mathrm{~cm}$ mentre il crollo non è evitabile per spessori inferiori.

$\mathrm{Nel}$ caso in cui si realizzi una finestra (o più finestre) in luogo di una o più delle porte esistenti nello schema iniziale, corrispondentemente diminuisce l'altezza di calcolo dei maschi e, pertanto, la parete nel suo complesso resiste meglio sia in termini di coefficiente di sicurezza $v$ sia in termini di resistenza delle fasce di piano. Dunque, la risposta sismica di un edificio si può migliorare anche realizzando aperture più ampie di quelle esistenti a condizione però di chiudere qualcuna delle aperture esistenti o di ridurne le dimensioni.

Si osserva, inoltre, che le campate della fascia di piano sovrastanti le finestre presentano coefficienti di sicurezza $\xi$ e $\psi$ inferiori rispetto alle campate sovrastanti le porte. Ciò è da attribuirsi al fatto che, a parità di spostamento $\mathrm{d}$, i maschi più tozzi, caratterizzati da forze ultime $\mathrm{V}_{\mathrm{u} \text {,oriz }}$ per scorrimento sui letti di malta, assorbono sollecitazioni di taglio $\mathrm{V}_{\mathrm{i}}$ maggiori rispetto agli altri maschi che presentano forze ultime a flessione $\mathrm{V}_{\mathrm{u}, \text { flex }}$.
In generale, per pareti dotate di uniformità strutturale si osserva che le fasce di piano prossime ai bordi della parete presentano coefficienti di sicurezza $\xi$ e $\psi$ inferiori a quelli delle fasce che si trovano nella parte centrale della parete. Infatti, le prime risultano maggiormente sollecitate rispetto alle seconde, essendo i coefficienti di ripartizione pari ad 1 per i nodi di estremità del traverso e pari a 0,5 per i nodi interni.

In generale, fasce di piano aventi stesso rapporto altezza/larghezza $(2 \mathrm{r} / \mathrm{b})$ resistono in maniera differente al variare dell'altezza dei maschi murari e precisamente le fasce di minor altezza $2 \mathrm{r}$ presentano resistenza maggiore. In generale, infatti, a parità di spessore della fascia e di caratteristiche meccaniche della muratura, il taglio ed il momento resistenti dipendono dall'altezza della fascia. A tal proposito, i risultati ottenuti da alcune elaborazioni, svolte mantenendo costante la snellezza dei maschi e di conseguenza i valori di $\mathrm{V}_{\mathrm{i}}$ e $\mathrm{h}_{\mathrm{i}}$, hanno mostrato che, variando l'altezza delle fasce, diminuisce la loro resistenza fino alla rottura che avviene per il valore più basso del rapporto $2 \mathrm{r} / \mathrm{b}$ mentre la resistenza della parete nel suo complesso non varia poiché lo schema strutturale è rimasto invariato.

Anche il comportamento di due pareti aventi uguale rapporto $2 \mathrm{r} / \mathrm{b}$ della fascia di piano, al variare di $\mathrm{r}$ e b, ed uguale snellezza dei maschi, mostra coefficienti $\xi$ e $\psi$ differenti poiché le sollecitazioni sulla fascia $\left(\mathrm{T}_{\mathrm{f}}\right.$ e $\mathrm{M}_{\mathrm{f}}$ ) non variano proporzionalmente al taglio ed al momento resistenti. Pertanto, il rapporto $2 \mathrm{r} / \mathrm{b}$ non è determinante al fine di definire la resistenza della fascia così come lo è il rapporto $\mathrm{h} / \mathrm{d}$ per i maschi.

Nelle elaborazioni eseguite considerando i principali schemi di pareti di fabbricati agricoli tradizionali (fig. 3 ), con spessori costanti della parete pari a $30 \mathrm{~cm}$, le altezze della fascia di piano comprese tra $50 \mathrm{~cm}$ e $70 \mathrm{~cm}$ rappresentano valori di soglia al di sotto dei quali le fasce sono soggette a rottura per taglio e/o momento.

Seguendo quanto descritto al punto 5.4.6.2.5 della normativa vigente [8] si ottengono valori minori per il taglio resistente e valori maggiori per il momento resistente rispetto a quelli calcolati secondo alcuni $\mathrm{Au}-$ tori [6]. In particolare, i valori del momento resistente forniti dalla norma sono più realistici in presenza di elementi orizzontali dotati di resistenza a trazione (catene o cordoli). Tuttavia, per i tipi di pareti esistenti esaminati (fig. 3) l'adozione del valore del taglio resistente secondo normativa conduce a risultati poco realistici poiché le fasce si romperebbero anche per spessori di $90 \mathrm{~cm}$ e altezze tra $0,3 \mathrm{~m}$ e $2,5 \mathrm{~m}$.

Per i diversi schemi strutturali esaminati riportati in fig. 3 si riportano nel seguito i diagrammi (figg. 4, $5,6,7,8,9)$ della forza sismica $F_{s}$ in relazione alla larghezza dei maschi $\mathrm{D}_{\mathrm{m}}$ al variare del parametro $t$ indicante lo spessore della muratura.

La fig. 4 si riferisce alla parete di tipo 1 in cui $r_{i}$ è pari a $125 \mathrm{~cm}$, rappresentativo del valore massimo riscontrato nelle tipologie edilizie esaminate, e l'altezza delle finestre $h_{f}$ è di $100 \mathrm{~cm}$. In tal caso le fasce rimangono integre e la forza sismica $\mathrm{F}_{\mathrm{s}}$ varia tra $16,3 \mathrm{t}$ e $148,7 \mathrm{t}$ con coefficienti di sicurezza $v$ compresi tra 


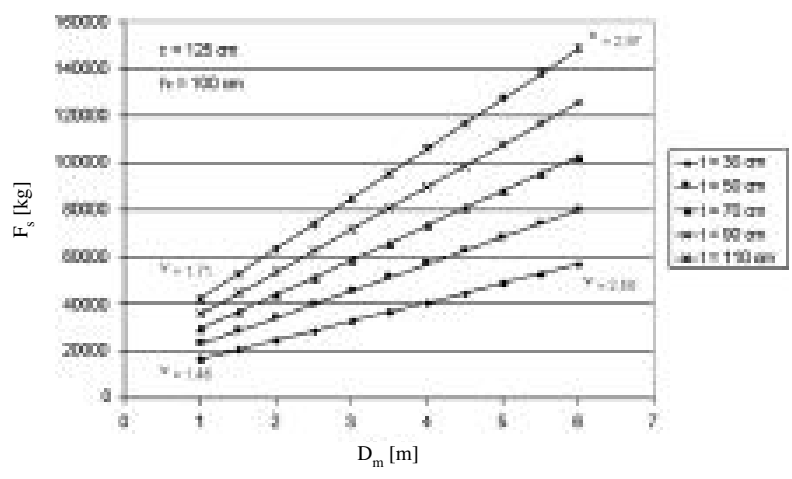

Fig. 4 - Diagramma della $F_{s}$ in funzione di $D_{m}$ al variare del parametro t, per parete di tipo 1 con $r_{i}=125 \mathrm{~cm} \mathrm{e} h_{f}=100 \mathrm{~cm}$, fissati $\sigma_{k}=200 \mathrm{tm}^{-2}$ e $\tau_{k}=7 \mathrm{tm}^{-2}$.

Fig. 4 - Chart of $\mathrm{F}_{\mathrm{s}}$ variation in relation to $\mathrm{D}_{\mathrm{m}}$ in dependence of the parameter $\mathrm{t}$, for wall type 1 with $r_{i}=125 \mathrm{~cm}$ and $\mathrm{h}_{\mathrm{f}}=100 \mathrm{~cm}$, with $\sigma_{\mathrm{k}}=200 \mathrm{tm}^{-2}$ e $\tau_{\mathrm{k}}=7 \mathrm{tm}^{-2}$.

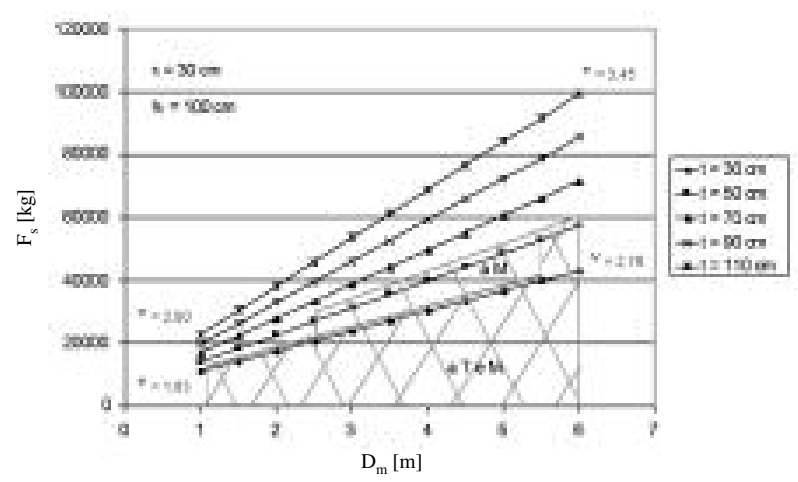

Fig. 5 - Diagramma della $F_{s}$ in funzione di $D_{m}$ al variare del parametro t, per parete di tipo 1 con $r_{i}$ pari a $30 \mathrm{~cm}$ e $h_{f}$ di 100 $\mathrm{cm}$, fissati $\sigma_{k}=200 \mathrm{tm}^{-2}$ e $\tau_{k}=7 \mathrm{tm}^{-2}$. Tratteggio singolo $=$ condizioni di crisi delle fasce dovuta a momento flettente; Tratteggio incrociato = condizioni di crisi delle fasce dovuta a sforzo di taglio e a momento flettente.

Fig. 5 - Chart of $\mathrm{F}_{\mathrm{s}}$ variation in relation to $\mathrm{D}_{\mathrm{m}}$ in dependence of the parameter $\mathrm{t}$, for wall type 1 with $r_{i}=30 \mathrm{~cm}$ and $\mathrm{h}_{\mathrm{f}}=100 \mathrm{~cm}$, with $\sigma_{\mathrm{k}}=200$ $\mathrm{tm}^{-2} \mathrm{e} \tau_{\mathrm{k}}=7 \mathrm{tm}^{-2}$. Single dashed line $=$ floor strip failure due to bending; Cross dashed line $=$ floor strip failure due to shear and bending.

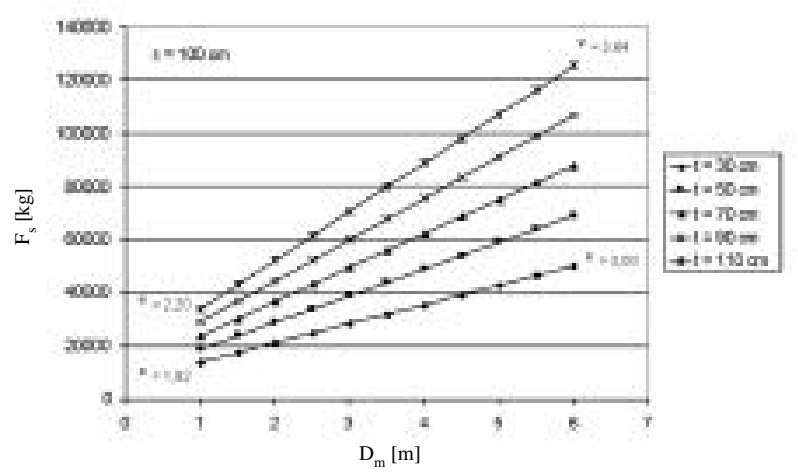

Fig. 6 - Diagramma della $F_{s}$ in funzione di $D_{m}$ al variare del parametro t, per parete di tipo 2 con $r_{i}$ pari a $100 \mathrm{~cm}$ e apertura centrale avente larghezza di $1,40 \mathrm{~m}$ altezza di $3,0 \mathrm{~m}$, fissati $\sigma_{k}=200 \mathrm{tm}^{-2}$ e $\tau_{k}=7 \mathrm{tm}^{-2}$.

Fig. 6 - Chart of $\mathrm{F}_{\mathrm{s}}$ variation in relation to $\mathrm{D}_{\mathrm{m}}$ in dependence of the parameter $\mathrm{t}$, for wall type 2 with $r_{i}=100 \mathrm{~cm}$ and middle opening of $1,40 \mathrm{~m}$ width and $3,0 \mathrm{~m}$ height, with $\sigma_{\mathrm{k}}=200 \mathrm{tm}^{-2} \mathrm{e} \tau_{\mathrm{k}}=7 \mathrm{tm}^{-2}$.

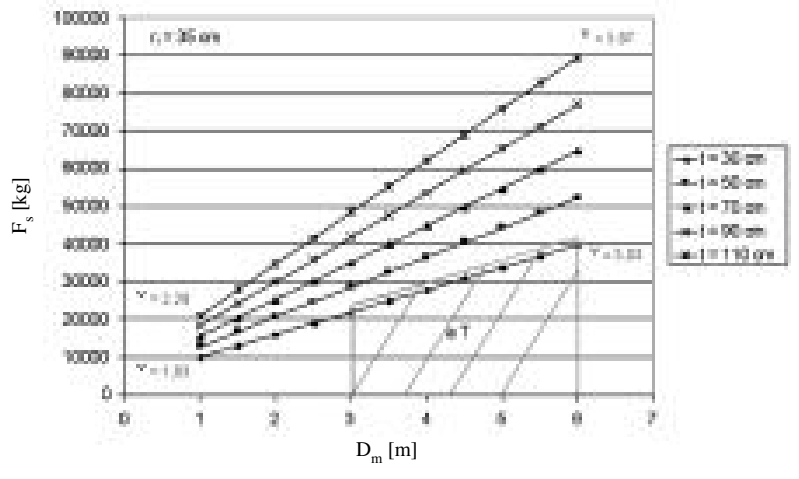

Fig. 7 - Diagramma della $F_{s}$ in funzione di $D_{m}$ al variare del parametro $t$, per parete di tipo 2 con $r_{i}=35 \mathrm{~cm}$ e apertura centrale avente larghezza di $1,40 \mathrm{~m}$ altezza di $3,00 \mathrm{~m}$, fissati $\sigma_{k}=200 \mathrm{tm}^{-2} e$ $\tau_{k}=7 \mathrm{tm}^{-2}$. Tratteggio singolo $=$ condizioni di crisi delle fasce dovuta a sforzo di taglio.

Fig. 7 - Chart of $\mathrm{F}_{\mathrm{s}}$ variation in relation to $\mathrm{D}_{\mathrm{m}}$ in dependence of the parameter $\mathrm{t}$, for wall type 2 with $r_{i}=35 \mathrm{~cm}$ and middle opening of 1,40 $\mathrm{m}$ width and $3,0 \mathrm{~m}$ height, with $\sigma_{\mathrm{k}}=200 \mathrm{tm}^{-2} \mathrm{e} \tau_{\mathrm{k}}=7 \mathrm{tm}^{-2}$. Single dashed line $=$ floor strip failure due to shear.

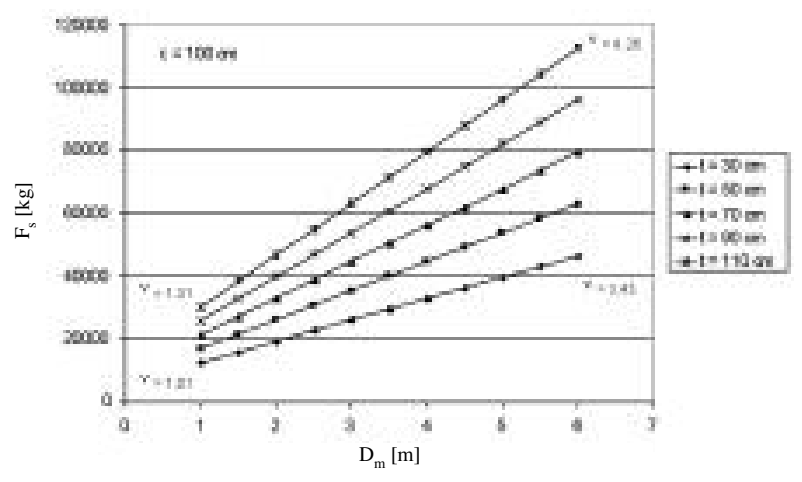

Fig. 8 - Diagramma della $F$ in funzione di $D_{m}$ al variare del parametro $t$, per parete di tipo 3 con $r_{i}$ pari a $100 \mathrm{~cm}$, fissati $\sigma_{k}=200 \mathrm{tm}^{-2}$ e $\tau_{k}=7 \mathrm{tm}^{-2}$.

Fig. 8 - Chart of $\mathrm{F}_{\mathrm{s}}$ variation in relation to $\mathrm{D}_{\mathrm{m}}$ in dependence of the parameter $\mathrm{t}$, for wall type 3 with $r_{i}=100 \mathrm{~cm}$, with $\sigma_{\mathrm{k}}=200 \mathrm{tm}^{-2} \mathrm{e}$ $\tau_{\mathrm{k}}=7 \mathrm{tm}^{-2}$.

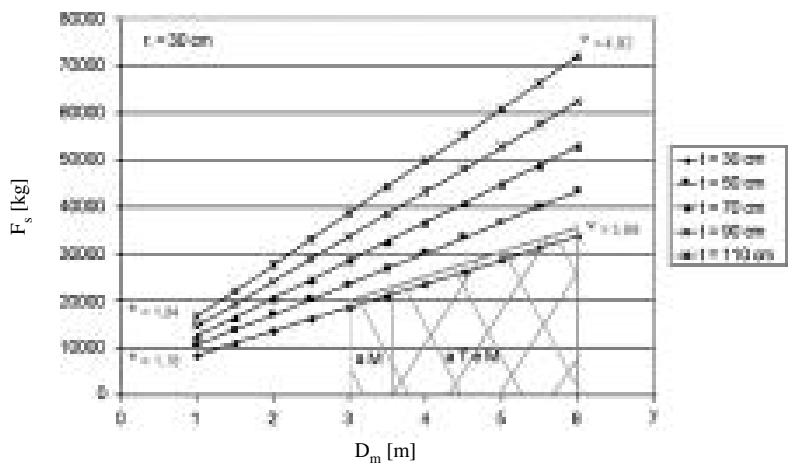

Fig. 9 - Diagramma della $F_{s}$ in funzione di $D_{m}$ al variare del parametro t, per parete di tipo 3 con $r_{i}$ pari a $30 \mathrm{~cm}$, fissati $\sigma_{k}=200 \mathrm{tm}^{-2}$ e $\tau_{k}=7 \mathrm{tm}^{-2}$. Tratteggio singolo $=$ condizioni di crisi delle fasce dovuta a momento flettente; Tratteggio incrociato $=$ condizioni di crisi delle fasce dovuta a sforzo di taglio e a momento flettente.

Fig. 9 - Chart of $\mathrm{F}_{\mathrm{s}}$ variation in relation to $\mathrm{D}_{\mathrm{m}}$ in dependence of the parameter $\mathrm{t}$, for wall type 3 with $r_{i}=30 \mathrm{~cm}$, with $\sigma_{\mathrm{k}}=200 \mathrm{tm}^{-2}$ e $\tau_{\mathrm{k}}=7$ $\mathrm{tm}^{-2}$. Single dashed line $=$ floor strip failure due to bending; Cross dashed line $=$ floor strip failure due to shear and bending. 
1,46 e 2,97, al variare dello spessore della parete e della larghezza dei maschi murari.

Il diagramma di fig. 4, così come risulta per gli altri diagrammi riportati nel presente lavoro, è valido strettamente per le caratteristiche geometriche $r_{i}$ ed $h_{f}$ prefissate. Infatti, se si incrementa l'altezza delle finestre sino a $h_{f}=200 \mathrm{~cm}$ i risultati ottenibili differiscono dal caso esaminato nella fig. 4 riferito alla condizione $\mathrm{h}_{\mathrm{f}}=100 \mathrm{~cm}$. In particolare, in corrispondenza dei medesimi valori di $\mathrm{F}_{\mathrm{s}}$, si ottengono valori del coefficiente di sicurezza n (compresi tra 1,30 e 3,92) che sono minori del caso con $h_{f}=100 \mathrm{~cm}$ se la snellezza dei maschi aumenta oltre il limite di 1,5 cui si associa $\mathrm{V}_{\text {uflex }}$, mentre sono maggiori se la loro snellezza rimane al di sotto della snellezza limite. Quest'ultima condizione determina valori minori dei coefficienti di sicurezza $\xi$ e $\psi$ delle fasce, dovuti alla maggiore sollecitazione di taglio trasmessa dai ritti, tanto che le fasce di estremità si rompono per taglio per uno spessore della muratura di $30 \mathrm{~cm}$ e larghezze dei maschi superiori a $2 \mathrm{~m}$.

La fig. 5 descrive la risposta della parete di tipo 1 in cui $r_{j}$ è pari a $30 \mathrm{~cm}$ e l'altezza delle finestre è di 100 $\mathrm{cm}$. Tale condizione è stata selezionata tra quelle elaborate perché rappresenta lo stato limite in cui le fasce di estremità della parete cominciano a rompersi a taglio e/o a momento, in corrispondenza di spessori della parete di $30 \mathrm{~cm}$ per i valori di $\mathrm{D}_{\mathrm{m}}$ considerati e di spessori di $50 \mathrm{~cm}$ per valori di $\mathrm{D}_{\mathrm{m}}$ maggiori di $2,5 \mathrm{~m}$. Infatti, per valori decrescenti di $r_{i}$ inferiori a $30 \mathrm{~cm}$ aumenta progressivamente il numero delle fasce di piano che pervengono a rottura, anche all'aumentare dello spessore $t$ della parete. Invece, per valori di $r_{i}$ maggiori di $30 \mathrm{~cm}$ le fasce rimangono integre lungo tutto il percorso di carico e in corrispondenza di tutti $\mathrm{i}$ valori di t e $\mathrm{D}_{\mathrm{m}}$ considerati.

La parete di tipo 1 in cui l'altezza delle finestre è $h_{\mathrm{f}}=200 \mathrm{~cm}$ differisce del caso con $h_{\mathrm{f}}=100 \mathrm{~cm} \mathrm{e} \mathrm{r}_{\mathrm{i}}=30$ $\mathrm{cm}$, poiché in corrispondenza dei medesimi valori di $\mathrm{F}_{\mathrm{s}}$ si ottengono valori del coefficiente di sicurezza $v$ (compresi tra 1,27 e 4,07 ) che sono minori se la snellezza dei maschi aumenta oltre il limite di 1,5 cui si associa $\mathrm{V}_{\text {uflex }}$, mentre sono maggiori se la loro snellezza rimane al di sotto della snellezza limite. Quest'ultima condizione determina valori minori dei coefficienti di sicurezza $\xi$ e $\psi$ delle fasce, dovuti alla maggiore sollecitazione di taglio trasmessa dai ritti, tanto che, per $\mathrm{t}=30$ $\mathrm{cm}$, tutte le fasce di piano della parete si rompono progressivamente da quelle di estremità verso quelle centrali, sia a taglio che a momento al crescere della larghezza $D_{m}$ dei maschi. Le fasce di estremità, inoltre, si rompono a taglio e momento per tutti i valori di t considerati. In tal caso, pertanto, occorrerà procedere ad un consolidamento delle fasce di estremità della parete.

La fig. 6 descrive il comportamento della parete di tipo 2 in cui $r_{i}$ è pari a $100 \mathrm{~cm}$, rappresentativo del valore massimo riscontrato nelle tipologie edilizie esaminate, e l'apertura centrale ha una larghezza di $1,40 \mathrm{~m}$ e altezza di $3,0 \mathrm{~m}$. In tal caso le fasce rimangono integre e la forza sismica varia tra 13,9 t e 125,6 $\mathrm{t}$ con coefficienti di sicurezza compresi tra 1,82 e
3,64 , in relazione allo spessore della parete ed alla larghezza dei maschi murari.

La parete di tipo 2 con apertura centrale di $1,20 \mathrm{~m}$ di larghezza e 2,40 $\mathrm{m}$ di altezza differisce dal precedente caso, oltre che per valori maggiori della $\mathrm{F}_{\mathrm{s}}$ (compresi tra 13,2 t e 114,2 t) e più elevati valori di $\mathrm{v}$ (compresi tra 1,98 e 3,98), per valori maggiori dei coefficienti di sicurezza $\xi$ e $\psi$ delle fasce, dovuti alla minore sollecitazione di taglio trasmessa dai ritti.

La fig. 7 descrive la risposta della parete di tipo 2 in cui $\mathrm{r}_{\mathrm{i}}$ è pari a $35 \mathrm{~cm}$ e l'apertura centrale è più ampia (1,40 m di larghezza e 3,00 m di altezza). Tale combinazione dimensionale è stata selezionata tra quelle elaborate perché rappresenta lo condizione in cui le fasce di estremità della parete cominciano a rompersi a taglio e/o a momento. In particolare, dal diagramma si osserva che le fasce di estremità iniziano a rompersi per $\mathrm{D}_{\mathrm{m}}>3 \mathrm{~m}$. La forza sismica varia tra 10,2 t e 89,6 t con coefficienti di sicurezza $v$ compresi tra 1,83 e 3,87 , in relazione allo spessore della parete ed alla larghezza dei maschi murari.

La parete di tipo 2 con apertura centrale meno ampia (1,20 m di larghezza e 2,40 $\mathrm{m}$ di altezza) presenta rottura delle fasce per $r_{i} \leq 25 \mathrm{~cm}$. In tal caso, ad $F_{s}$ minori del caso con apertura centrale più ampia (comprese tra 8,5 t e 69,7 t) ed a valori di $v$ più elevati (compresi tra 2,11 e 4,64) sono associate $F_{u}$ minori dovute al minor peso proprio del muro, e le fasce di estremità, per $\mathrm{t}=30 \mathrm{~cm}$, si rompono a momento per $\mathrm{D}_{\mathrm{m}} \geq 1 \mathrm{~m}$ ed a taglio per $\mathrm{D}_{\mathrm{m}} \geq 3,5 \mathrm{~m}$.

La fig. 8 si riferisce alla parete di tipo 3 in cui $r_{i}$ $=100 \mathrm{~cm}$. In tal caso le fasce sono integre e la forza sismica $F_{s}$ varia tra $12,6 \mathrm{t}$ e $112,6 \mathrm{t}$ con coefficienti di sicurezza $v$ compresi tra 1,01 e 4,26 , in relazione allo spessore della parete ed alla larghezza dei maschi murari.

La parete di tipo 3 con $r_{i}=30 \mathrm{~cm}$ (fig. 9) mostra, in corrispondenza di forze sismiche $\mathrm{F}_{\mathrm{s}}$ minori del caso precedente (comprese tra 8,4 t e 71,9 t) e coefficienti di sicurezza $v$ poco più elevati (compresi tra $1,18 \mathrm{e}$ 4,92 ), una minore resistenza delle fasce che, per $\mathrm{t}=30$ $\mathrm{cm}$, si rompono a momento per $\mathrm{D}_{\mathrm{m}} \geq 3 \mathrm{~m}$ e a taglio per $\mathrm{D}_{\mathrm{m}} \geq 3,5 \mathrm{~m}$.

Nei diagrammi descritti è stata posta maggior attenzione all'analisi del comportamento delle fasce di piano in relazione alla resistenza complessiva delle pareti esaminate. Tuttavia, al variare della geometria della parete e delle sue caratteristiche di resistenza, il modello proposto consente, inoltre, di analizzare:

- il tipo di meccanismo di collasso proprio di ciascun maschio murario (fessurazione diagonale, scorrimento lungo i letti di malta, flessione);

- lo stato integro o fessurato della muratura durante il percorso di carico, mediante la definizione delle curve reologiche dei maschi murari ed il calcolo della resistenza ultima del traverso;

- la resistenza delle fasce di piano, verificandone l'integrità e la necessità di consolidamento in relazione alle proprie caratteristiche geometriche e di resistenza. 


\section{CONCLUSIONI}

\section{Conclusions}

Nel presente lavoro è stato proposto un metodo di verifica sismica di pareti in muratura portante nel caso di azioni complanari alle pareti stesse, appropriato per le tipologie "specialistiche" dei fabbricati agricoli tradizionali.

L'applicabilità del metodo di calcolo proposto è stata verificata studiando il comportamento statico di alcuni tipi di pareti al variare di taluni parametri geometrici e delle caratteristiche di resistenza della muratura.

L'utilizzo dei diagrammi consente di trovare il valore della forza sismica cui la parete è capace di resistere, in funzione della larghezza dei maschi murari (e quindi della loro snellezza) e dello spessore della muratura.

I diagrammi prodotti possono risultare utili anche ai fini di una verifica preliminare di pareti aventi caratteristiche geometriche simili a quelle esaminate.

In particolare, nel caso in cui si voglia verificare l'integrità delle fasce di piano, i diagrammi possono fornire indicazioni per i casi in cui la snellezza di uno o più maschi sia minore di quella dei corrispondenti maschi dello schema scelto. In tal caso, si avranno valori più contenuti del taglio trasmesso dai ritti alle fasce e quindi è assicurato il livello di integrità delle fasce indicato dal diagramma.

Ulteriori approfondimenti potranno riguardare sia la scelta dei parametri caratteristici di resistenza della muratura $\left(\tau_{\mathrm{k}}, \sigma_{\mathrm{k}}, \sigma_{\mathrm{fp}}, \tau_{\mathrm{kf}}\right)$, dato che allo stato attuale non si hanno a disposizione sperimentazioni su muratura lavica di fabbricati agricoli d'esercizio tradizionali dell'area etnea né, tantomeno, su murature laviche consolidate, sia la verifica di schemi strutturali relativi a costruzioni realmente esistenti.

\section{BIBLIOGRAFIA}

\section{References}

[1] Binda L., Penazzi D., Mirabella Roberti G., BaRONIO G., Tedeschi, Tiraboschi C. (1999), Indagini per la caratterizzazione dei materiali. $9^{\circ}$ Convegno Nazionale "L'ingegneria sismica in Italia", Torino, 20-23 Ottobre 1999.

[2] Braga F., Dolce M., A method for the analysis of antiseismic masonry multistorey buildings, Proceedings of the Sixth International Brick Masonry Conference, Roma, 1982, pp. 1089-1099.

[3] Cascone G., Di Fazio S., Arcidiacono C., La verifica di sicurezza nel riuso dei fabbricati agricoli tradizionali in zona sismica, Atti del IV Seminario della Seconda Sezione dell'AIGR su: 'Il recupero dell'edilizia rurale nel contesto territoriale', vol. 3, Sassari, 13-16 giugno 1994, pp. 735-746.

[4] Cascone G., Di Fazio S., ARCidiacono C., Il recupero degli edifici rurali tradizionali in zona sismica. Materiali, tecniche costruttive e tipi di intervento, Atti del IV Seminario della Seconda Sezione dell'AIGR su:'Il recupero dell'edilizia rurale nel contesto territoriale', vol. 3, Sassari, 13-16 giugno 1994, pp. 723-734.

[5] Cuomo M., Badalà A., Problematiche metodologiche relative alla determinazione sperimentale delle proprietà meccaniche dei materiali murari e dei loro componenti. In "La protezione del patrimonio culturale - La questione sismica" II Seminario Nazionale di studio, Roma, 9-10 aprile 1997: 657-683, Roma, (1998): Gangemi Editore.

[6] Fusier F., Vignoli A., Proposta di un metodo di calcolo per edifici in muratura sottoposti ad azioni orizzontali, Ingegneria sismica (1993), Anno X (1), 10-24.

[7] Gazzetta Ufficiale della Repubblica italiana, Norme tecniche per le costruzioni in zone sismiche, D.M. 16/01/1996, G.U. n. 29 del 05/02/1996, Roma.

[8] Gazzetta Ufficiale della Repubblica italiana, Norme tecniche per le costruzioni, D.M. 14/09/2005, G.U. n. 222 del 23/09/2005 Suppl.ord.n.159. Roma.

[9] Liberatore (a cura di), Progetto Catania: indagine sulla risposta sismica di due edifici in muratura, CNR-GNDT - Roma, 2000, 275 pp.

[10] Ministero dei LL.PP. Legge 14 maggio 1981 n. 219, Istruzioni per l'applicazione della normativa tecnica per la riparazione ed il rafforzamento degli edifici danneggiati dal sisma.

[11] Mотта F., Resistenza sismica degli edifici in muratura, Ingegneria Sismica (1992), Anno IX, N.3, 3-14.

[12] Ord. Prot. Civ. n.3274/2003, Primi elementi in materia di criteri generali per la classificazione sismica del territorio nazionale e di normative tecniche per le costruzioni in zona sismica, Presidenza del Consiglio dei Ministri. Roma.

[13] Palumbo G., Le residenze di campagna nel versante orientale dell'Etna, 1991, Documenti D.A.U. Dipartimento di Architettura e Urbanistica n.6, Università degli Studi di Catania.

[14] Pozzati P., Teoria e tecnica delle strutture, UTET, Torino (1972).

Parole chiave: Muratura lavica, Verifica sismica, Edifici agricoli tradizionali.

\section{SUMMARY}

\section{DEFINITION OF A SAFETY VERIFICATION METHOD FOR IN-PLANE SEISMIC FORCES OF TRADITIONAL RURAL BUILDINGS WALLS}

In this paper a method for the seismic safety verification for in-plane actions, was applied for the analysis of building walls of traditional rural buildings of the Etna area, made of lava stones. The behaviour of some wall typologies of these buildings were analyzed in dependence of several geometric and resistance characteristics.

The results are presented as diagrams that, by relating seismic force with wall width and thickness, could be a useful tool to perform a preliminary verification of this walls and to support wall design for the same typology of buildings.

Key words: Masonry walls, Safety verification, traditional rural buildings. 